\title{
雨
}

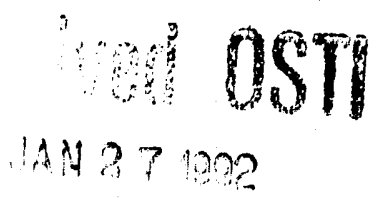

\section{Squeezing Dip Moveout for Depth-Variable Velocity}

by

\author{
Dave Hale and Craig Artley
}

\section{DISCLAIMER}

account of work sponsored by an agency of the United States Neither Gnvernment nor any agency thereof, nor any of their Government. Neither the United States Gnernes implied, or assumes any legal liability or responsiemployees, makes any warranty, express or implied, of any information, apparatus, product, or bility for the accuracy, completeness, of use would not infringe privately owned rights. Referprocess disclosed, or represents that its use would process, or service by trade name, trademark, ence herein to any specific commercial product, procosstitute or imply its endorsement, recommanufacturer, or otherwise does not necessarily constituent or any agency thereof. The views mendation, or favoring by the United States Governmecessarily state or reflect those of the

and opinions of authors expressed herein do not
United States Goversment or any agency thereof.

\section{Center for Wave Phenomena \\ Colorado School of Mines \\ Golden, Colorado 80401}

(303)273-3557 


\title{
Squeezing dip moveout for depth-variable velocity
}

\author{
Dave Hale and Craig Artley
}

\begin{abstract}
Iii dip moveout (DMO) processing, velocity variations with depth can be handled approximately by squeezing a constant-velocity DMO operator to narrow its impulse response. This squeezed DMO approximation provides a computationally efficient and reasonably accurate method of DMO correction for depth-variable velocity.

DMO is squeezed by two modifications to constant-velocity DMO. One modification is a squeezing function of time that depends only on simple time averages of velocity that are likely to be known before DMO is applied. This squeeze function ensures that squeezed DMO accurately handles moderately steep reflections, and can be incorporated with simple time stretching before and after DMO, withont any changes to existing constant-velocity DMO methods. The second modificition is a constant squeezing factor, which may be used to tune squeczed DMO) to better handle steep reflections. This factor requires only trivial changes to constant-velocity DMO methods.

Tests with both synthetic and recorded seismic data suggest that squeezed $\mathrm{DMO}$ is an effective method for handling velocity variations with depth. These tests also show that the differences between constant-velocity DMO and squeczed DMO caii be significant.
\end{abstract}

\section{INTRODUCTION}

Although velocity variations are routinely honored in normal moveout (NMO) and migration processing of seismic data, velocity variations are often ignored in dip moveout (DMO) processing. Constant-velocity DMO processing is often used in arcas where velocity is known to increase significantly with depth.

One reason for the popularity of constant-velocity DMO may be that precise mothods for handling velocity variations in DMO processing are computationally inefficient or difficult to implement. Another may be that the crross in constant-velority D.M() 
processing are less significant than other errors in seismic data processing. Furthermore, Lynn et al. (1991) suggest that errors in assuming constant velocity in DMO processing may, in some areas, compensate for errors due to assuming isotropic wave propagation.

Effects such as anisotropy are today significant, in part, because of increasing demands on the precision of seismic imaging. To improve the precision of DMO processing, several authors (e.g., Artley, 1991; Meinardus and Schleicher, 1991; Witte, 1991) have described methods for precise DMO correction where velocity varies with depth. However, these algorithms for exactly handling depth-variable velocity tend to be less efficient and more complex than their constant-velocity counterparts. Constantvelocity DMO is a relatively simple process, and may be performed with a variety of computationally efficient algorithms (e.g., Biondi and Ronen, 1987; Notfors and Godfrey, 1987; Barry and Drecun, 1989; Hale, 1991).

In this paper, we describe a method for approximately handling velocity variations with depth through simple and efficient modifications to constant-velocity DMO. Briefly, we derive a time-variable scaling function that we use to squeeze a constantvelocity DMO operator so that it approximates an exact DMO operator for a wide range of offsets and reflector dips. This approach was first proposed by Rocca (1982, personal communication), was developed by Rocca and others (Hale, 1983, 1988; Bolondi and Rocca, 1985; Deregowski, 1985, 1987), and has been used in various forms in the seismic processing industry. In this paper, we summarize and enhance this approach, and demonstrate its effectiveness with applications to synthetic and recorded seismic data.

To illustrate the effect of squeezing DMO, we applied both corstant-velocity DMO and squeezed DMO to data recorded near a salt dome, and obtained the stacks shown in Figures $1 \mathrm{a}$ and $1 \mathrm{~b}$, respectively. Identical NMO velocities were used to obtain both stacks; the differences between the steep salt reflections of these two sections are due entirely to differences in DMO processing. Time-variable squeezing of the DMO operator has enabled steep reflections and horizontal reflections to be enhanced simultaneously by CMP stacking.

In the following section, we describe the squeezed DMO algorithm used to obtain the stack of Figure 1b, and show how time-variable squeezing can be incorporated easily into several popular constant-velocity DMO methods to approsimately handle velocity variations with depth. Then, we illustrate the effectiveness of this squeezed approximation with a synthetic data example and a more thorough discussion of tise recorded data example shown in Figure 1.

\section{SQUEEZING DMO}

The effect of constant-velocity DMO on reflection times is concisely summarized by the well-known DMO ellipse (e.g., Deregowski and Rocca, 1981; Deregowski, 1986):

$$
t_{0}=t_{n}\left(1-\frac{x^{2}}{h^{2}}\right)^{1 / 2}
$$




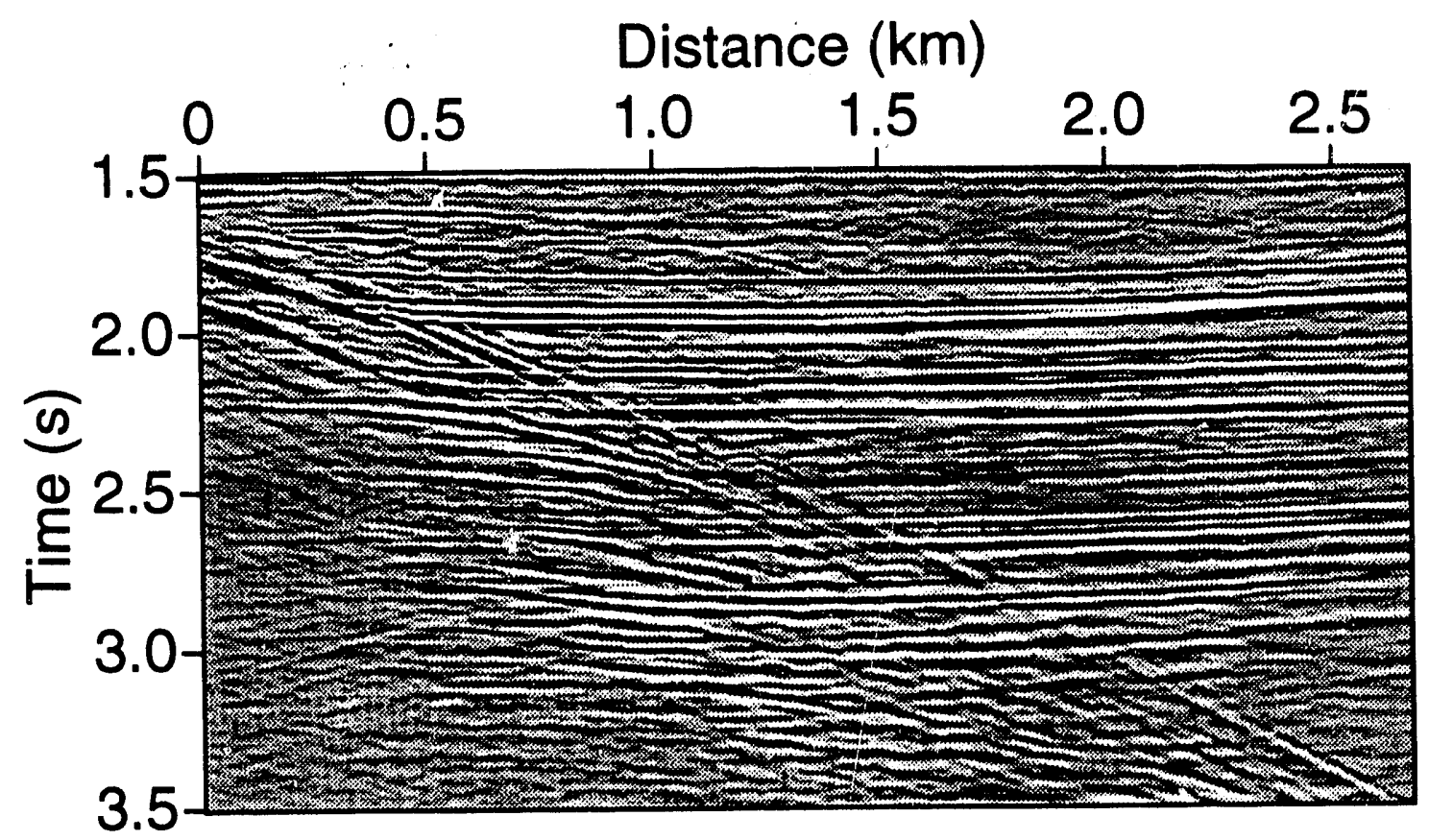

(a)

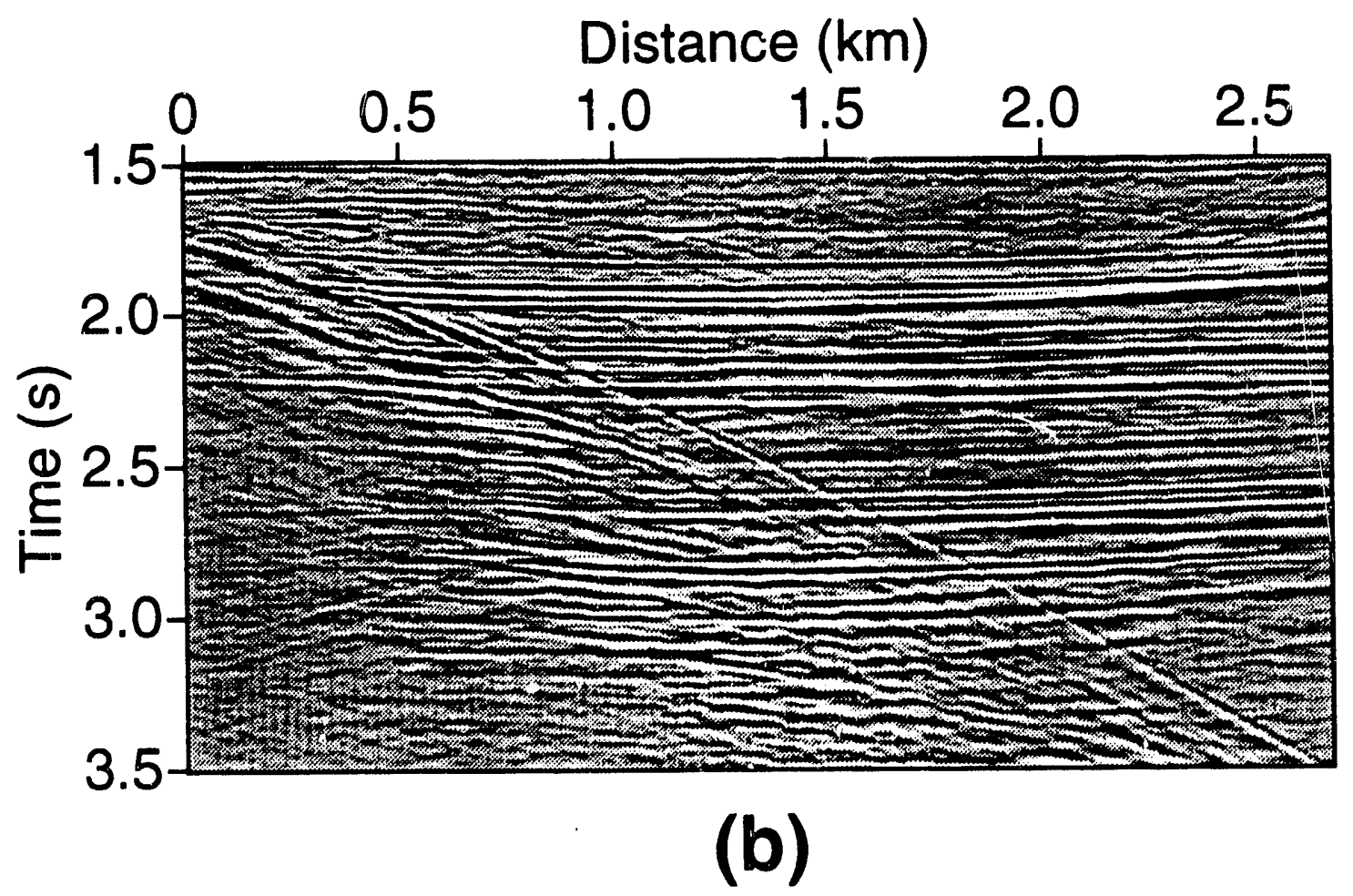

FIG. 1. Stacked sections near a salt dome with (a) constant-velocity DMO and (b) DMO squeezed to approximately handle velocity variations with depth. Squeezing DMO for depth-variable velocity enables horizontal reflections and the steep reflection off the salt dome to be enhanced with the same N.MO velocities. 
where $t_{0}$ is zero-offset time (after DMO), $t_{n}$ is NMO-corrected time (before DMO), $x$ is horizontal distance measured from the source-receiver midpoint, and $h$ is half the source-receiver offset. Some DMO methods ("Kirchhoff" or "integral" methods) use this expression directly to map each snmple of NMO-corrected seismic data to its appropriate zero-offset time $t_{0}$ and position $x$ before CMP stacking. Others methods, such as Fourier transform methods, perform the same mapping in a less direct way. We use the term constant-velocity DMO to refer to all of these methods.

To approximately handle velocity variations with depth, we propose the following squeezed version of the DMO mapping:

$$
t_{0}=t_{n}(1-S)+t_{n} S\left[1-\frac{x^{2}}{S \gamma\left(t_{n}\right) h^{2}}\right]^{1 / 2} .
$$

As derived by Hale $(1923,1988), \gamma(t)$ is a time-variable squeeze function defined by

$$
\gamma(t) \equiv \frac{3}{2} \frac{V_{4}^{4}(t)}{V_{2}^{4}(t)}-\frac{1}{2}-\frac{t}{V_{2}(t)} \frac{d V_{2}}{d t}
$$

where

$$
V_{2}(t) \equiv\left[\frac{1}{t} \int_{0}^{t} d s v^{2}(s)\right]^{1 / 2}
$$

and

$$
V_{4}(t) \equiv\left[\frac{1}{t} \int_{0}^{t} d s v^{4}(s)\right]^{1 / 4}
$$

are averages of the interval velocity function of time $v(t)$. The average $V_{2}(t)$ is the familiar root mean square (rms) velocity. We will refer to the constant factor $S$ in equation (2) as the DMO squeeze factor. Note that squeezed DMO becomes constantvelocity DMO for $\gamma(t)=S=1$.

\section{The effect of $\gamma(t)$ and $S$}

The effect of squeezing DMO via equation (2) is illustrated in Figure 2, for a subsurface in which velocity increases linearly with depth. The dotted curve plotted in Figure 2 represents the exact DMO mapping for this velocity function. (See, for example, Popovici, 1990, or Artley, 1991, for a discussion of this exact mapping.) The gray curve corresponds to the constant-velocity DMO mapping of equation (1), which is significantly different from the exact DMO mapping. The black curve corresponds to the squeezed DMO mapping of equation (2), and is a much better fit to the exact curve, at least up to the cusps in the exact mapping. For this example, the squeeze function $\gamma(t)$ was computed via equation (3) for the known velocity function, and the squeeze factor $S$ in equation (2) was set to 0.6.

From equation (2), it is easy to show that the curvature of the squeezed DMO mapping at its minimum (at $x=0$ ) is

$$
\left.\frac{\partial^{2} t_{0}}{\partial x^{2}}\right|_{x=0}=-\frac{t_{n}}{\gamma\left(t_{n}\right) h^{2}}
$$




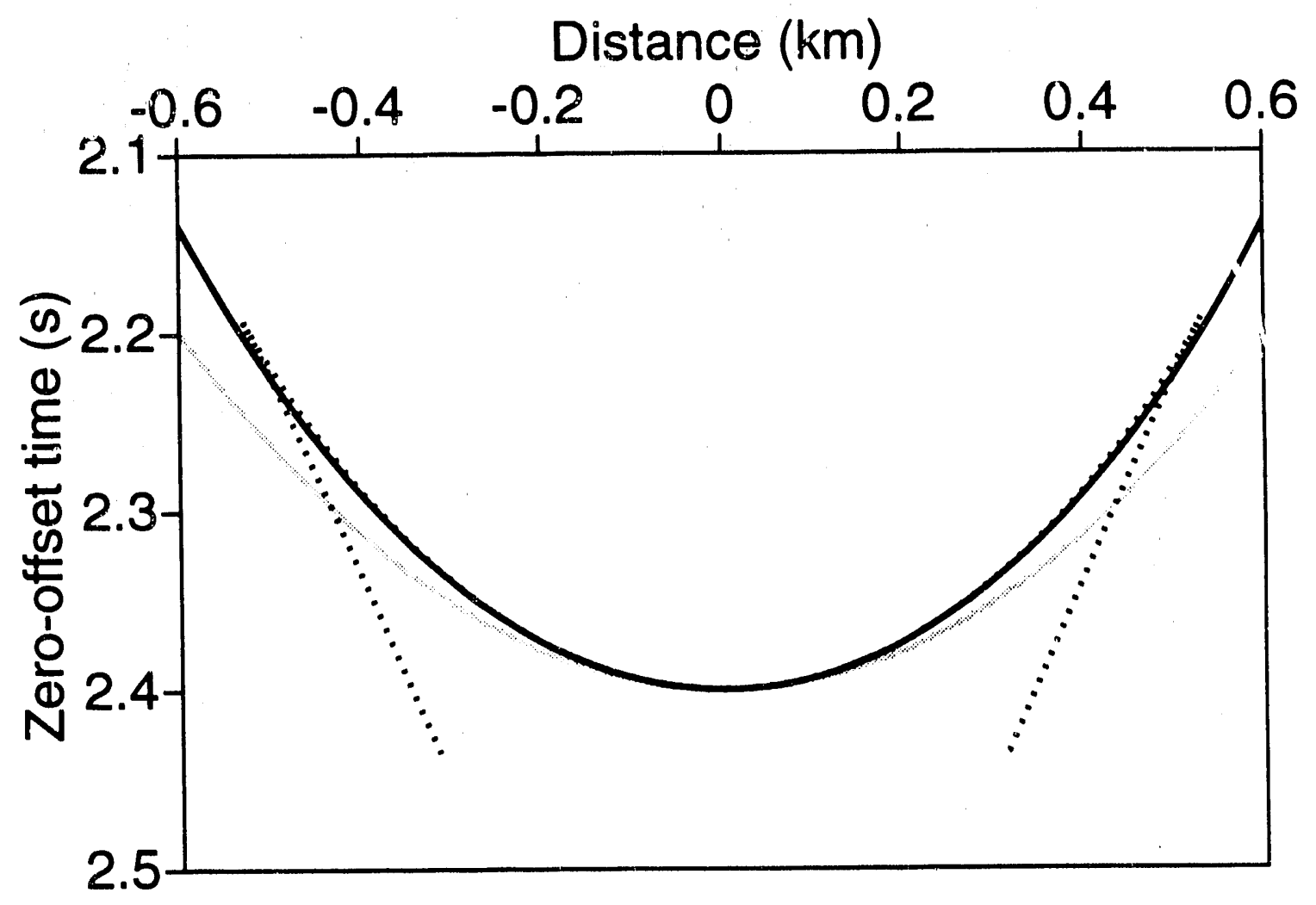

FIG. 2. Zero-offset time $t_{0}$ as a function of distance $x$, for NMO time $t_{n}=2.4 \mathrm{~s}$ and half-offset $h=1.5$. Times are plotted for three DMO methods: (gray) constantvelocity DMO, (black) squeezed DMO, and (dotted) exact DMO. Squeezed DMO closely approximates exact DMO up to the cusps in the exact curve. In this example, velocity $v$ varies with depth $z$ according to $v(z)=1.5+0.8 z$. 
which is independent of the squeeze factor $S$. The effect of the squeeze function $\gamma(t)$ given by equation (3) is to match the curvature of the exact DMO mapping at $x=0$.

For a typical increase in velocity with depth, we find that this curvature matching with $\gamma(t)$ yields a DMO mapping that is narrower than the constant-velocity DMO ellipse. To understand why, recall that the Cauchy-Schwarz inequality implies $V_{4}(t) \geq$ $V_{2}(t)$, so that the first two terms in equation (3) satisfy the inequality

$$
\frac{3}{2} \frac{V_{4}^{4}(t)}{V_{2}^{4}(t)}-\frac{1}{2} \geq 1,
$$

where equality occurs only when velocity is constant. The third term in equation (3), $-t / V_{2} \cdot d V_{2} / d t$, is negative for velocity increasing with depth, so its effect is to push the factor $\gamma(t)$ back towards unity. Therefore, $\gamma(t)$ could be less than or greater than unity, depending on which terms are dominant. However, because the first term consists of velocity averages over time $t$, the third term (with its velocity derivative) has a more immediate effect on $\gamma(t)$. For typical increases in velocity with depth, the third term dominates, and $\gamma(t)$ tends to be less than unity. This is why we refer to equation (2) as a "squeezed" rather than "stretched" DMO mapping.

The purpose of the squeeze factor $S$ is to improve the fit to the exact DMO mapping for the steeper slopes away from $x=0$. This factor is analogous to the so-called " $W$ factor" used to handle depth-variable velocity with constant-velocity Stolt migration (Stolt, 1978). For $S<1$, the effect of this factor is to further squeeze the DMO operator.

For typical velocity increases with depth, we have observed that the accuracy of squeezed DMO depends less on $S$ than on $\gamma(t)$. For the example of Figure 2, we found that choosing $S=0.4$ (result not shown) would make the squeezed DMO curve virtually identical to the exact DMO curve (up to the cusps in the exact curve). However, for typical velocity gradicnts (less than the gradient of $0.8 s^{-1}$ used here), we find that $S=0.6$ is often a reasonable choice, and the example in Figure 2 confirms that using $S=0.6$ instead of the more accurate $S=0.4$ yields an acceptable approximation.

\section{Squeezing DMO by Fourier transform}

The squeezed DMO mapping of equation (2) may be used directly in integral methods for DMO correction. A similar modification can be used to squeeze DMO by Fourier transform. For constant velocity, DMO may be performed by the following change of Fourier integration variable from NMO time $t_{n}$ to zero-offset time $t_{0}$ :

$$
t_{0}=t_{n}\left(1+\frac{k^{2} h^{2}}{\omega_{0}^{2} t_{n}^{2}}\right)^{1 / 2}
$$

(Hale, 1984), where $k$ is wavenumber and $\omega_{0}$ is zero-offset frequency. (The ratio $k / \omega_{0}$ is equivalent to reflection slope in a zero-offset section.) For depth variable velocity, 
we replace this mapping with

$$
t_{0}=t_{n}(1-S)+t_{n} S\left[1+\frac{\gamma\left(t_{n}\right) k^{2} h^{2}}{S \omega_{0}^{2} t_{n}^{2}}\right]^{1 / 2}
$$

Using the method of stationary phase (e.g., Liner, 1990), one can analytically evaluate the impulse response of squeezed DMO by Fourier transform implied by equation (5). The shape of that impulse response is approximately elliptical and is given by the squeezed DMO mapping of equation (2).

\section{Squeezing DMO via time stretching}

The simplest implementations of DMO by Fourier transform, based on either equation (4) or (5), are computationally inefficient. To improve the computational efficiency of DMO by Fourier transform, Notfors and Godfrey (1987) proposed a logarithmic time stretching. This stretching first maps NMO time $t_{n}$ to a stretched NMO time $\tau_{n}$ via the mapping

$$
\tau_{n}=T_{c} \ln \left(t_{n} / T_{c}\right),
$$

where $T_{c}^{\prime}$ is a time constant. After logarithmic time stretching, DMO can be approximated by an efficient multiplication in the frequency domain with the factor

$$
\exp \left\{i \omega_{\tau} T_{c}\left[\left(1+\frac{k^{2} h^{2}}{\omega_{\tau}^{2} T_{c}^{2}}\right)^{1 / 2}-1\right]\right\},
$$

where $\omega_{\tau}$ denotes stretched frequency corresponding to stretched time $\tau$. The resulting DMO-corrected data are then unstretched back to zero-offset time $t_{0}$. Liner (1990) provides a thorough discussion of this coistant-velocity DMO method, including an analysis of its accuracy.

Unfortunately, logarithmic time stretching is appropriate for constant-velocity DMO only. Therefore, we now derive a new (non-logarithmic) time stretching function that can be used before and after any constant-velocity DMO method to enable that method to approximately handle velocity variations with depth. For the particular method of DMO by Fourier transform, this new time stretching and the logarithmic time stretching can be combined to account for depth-variable velocity.

Recalling that the squeeze function $\gamma(t)$ in equation (2) was derived to match the curvature (second derivative) of the exact DMO time mapping at $x=0$, we seek a time stretching function that will enable constant-velocity DMO to produce this same curvature. For small $x$, we approximate the DMO mapping of equation (2) with

$$
t_{0} \approx t_{n}-\frac{t_{n} x^{2}}{2 \gamma\left(t_{n}\right) h^{2}} .
$$

Again, note that the squeeze factor $S$ does not appear in this approximation. We then seek a time stretching function $u(t)$ such that

$$
u\left(t_{0}\right) \approx u\left(t_{n}\right)-\frac{u\left(t_{n}\right) x^{2}}{2 h^{2}} .
$$


The motivation behind equation (9) is that, in the stretched time coordinate $u$, we can use constant-velocity DMO because the squeeze function $\gamma\left(t_{n}\right)$ does not appear in this equation.

Take the ratio of equations (9) and (8) to obtain

$$
\frac{u\left(t_{0}\right)-u\left(t_{n}\right)}{t_{0}-t_{n}} \approx \frac{u\left(t_{n}\right) \gamma\left(t_{n}\right)}{t_{n}} .
$$

For small $x, t_{0} \approx t_{n}$, and this equation becomes a differential equation for the time stretching function $u(t)$ :

or

$$
\frac{d u}{d t}=\frac{u \gamma(t)}{t}
$$

with solution

$$
\frac{d(\ln u)}{d t}=\frac{\gamma(t)}{t},
$$

$$
u(t)=T_{c} \exp \left[\int_{T_{c}}^{t} d s \frac{\gamma(s)}{s}\right] .
$$

For any velocity variation with depth, we can evaluate the squeeze function $\gamma(t)$, and then use equation (10) to evaluate the time stretching function $u(t)$. After stretching time with this function, we can use any constant-velocity DMO method, and afterwards unstretch time using the same function. The result of this stretching and unstretching is that we will have enabled constant-velocity DMO to approximately handle velocity variations with depth.

In the special case of DMO by Fourier transform, we may combine the new stretching defined by equation (10) and the logarithmic stretching defined by equation (6) to obtain

$$
\tau_{n}\left(t_{n}\right)=T_{c} \int_{T_{c}}^{t_{n}} d s \frac{\gamma(s)}{s} .
$$

For constant velocity, $\gamma(t)=1$, and this combined time stretching function becomes the logarithmic stretching function of equation (6).

Time stretching before and unstretching after constant-velocity DMO will produce a DMO operator with the correct curvature at $x=0$, but it does not account for the squeeze factor $S$ in equations (2) and (5). Recall that this factor does not affect the curvature at $x=0$, but that it enables the squeezed DMO operator to better approximate the exact DMO operator for steep reflection slopes away from $x=0$.

Fortunately, because we have assumed that the factor $S$ is constant, it can be easily incorporated into any constant-velocity $\mathrm{DMO}$ method. For example, after time stretching according to equation (11), we would modify Notfors and Godfrey's (1987) DMO method by replacing the phase-shifting factor in equation (7) with

$$
\exp \left\{i \omega_{\tau} T_{c} S\left[\left(1+\frac{k^{2} h^{2}}{S \omega_{\tau}^{2} T_{c}^{2}}\right)^{1 / 2}-1\right]\right\}
$$


This modification is trivial, and requires no significant increase in computational cost. Furthermore, this modification may be used even when velocity is constant to improve the accuracy of Notfors and Godfrey's approximation. As shown by Liner (1990), equation (7) yields an approximate DMO operator that is wider than the constant-velocity DMO ellipse. We found that using $S \approx 0.6$ in equation (12) all but eliminates the error in this approximation for constant-velocity DMO. Combining this factor of 0.6 with an additional factor of 0.6 to handle depth-variable velocity, we found empirically that $S \approx 0.6 \times 0.6 \approx 0.4$ provides a good approximation to the exact DMO operator when using Notfors and Godfrey's method with the time stretching of equation (11).

\section{SYNTHETIC DATA EXAMPLE}

To test squeezed DMO, and to empirically determine a reasonable value for the squeeze factor $S$, we applied squeezed DMO to synthetic seismograms computed for a subsurface model in which velocity increases linearly with depth $z$, according to $v(z)=1.5+0.8 z \mathrm{~km} / \mathrm{s}$. The model, shown in Figure 3, contains five reflectors, each with a dipping and horizontal segment. Dips for the dipping segments ranged from 30 to 90 degrees in 15 degree increments.

One of the CMP gathers from this synthetic seismic survey is plotted in Figure 4a. This CMP gather contains ten distinct events, five of which exhibit the normal moveout expected for horizontal reflectors. The other five events, including the last three events in this gather, which correspond to dips of 60,75 , and 90 degrees, exhibit the decreased moveout associated with dipping reflectors. Note that the last event in Figure 4a exhibits no moveout (no change in reflection time $w$ : th offset), as expected for a reflector dip of 90 degrees.

NMO correction of the CMP gather of Figure 4a, using the known rms velocity function $V_{2}(t)$, yields the CMP gather plotted in Figure $4 \mathrm{~b}$. Five of the events in this gather, those corresponding to horizontal reflectors, have been well aligned by NMO. As expected, the five events corresponding to the dipping reflectors have been over-corrected. A typical mute has been applied to zero events at early times and far offsets that are stretched significantly by NMO correction.

By applying constant-velocity DMO to the entire set of NMO-corrected synthetic traces (not just the CMP gather shown here), we obtained the CMP gather plotted in Figure 4c. In this example, constant-velocity DMO fails to align the reflections from the dipping reflectors, particularly for the steeper dips, corresponding to the last three events in this gather. In effect, constant-velocity DMO has gone too far in correcting for the errors in NMO correction seen in Figure 4b. Here, the increase in velocity with depth implies that we need less DMO correction than that provided by constant-velocity DMO.

Squeezed DMO, with $\gamma(t)<1$ computed according to equation (3), results in the CMP gather shown in Figure $4 \mathrm{~d}$. The alignment of reflections corresponding to dipping reflectors has been improved significantly, with only slight errors visible 


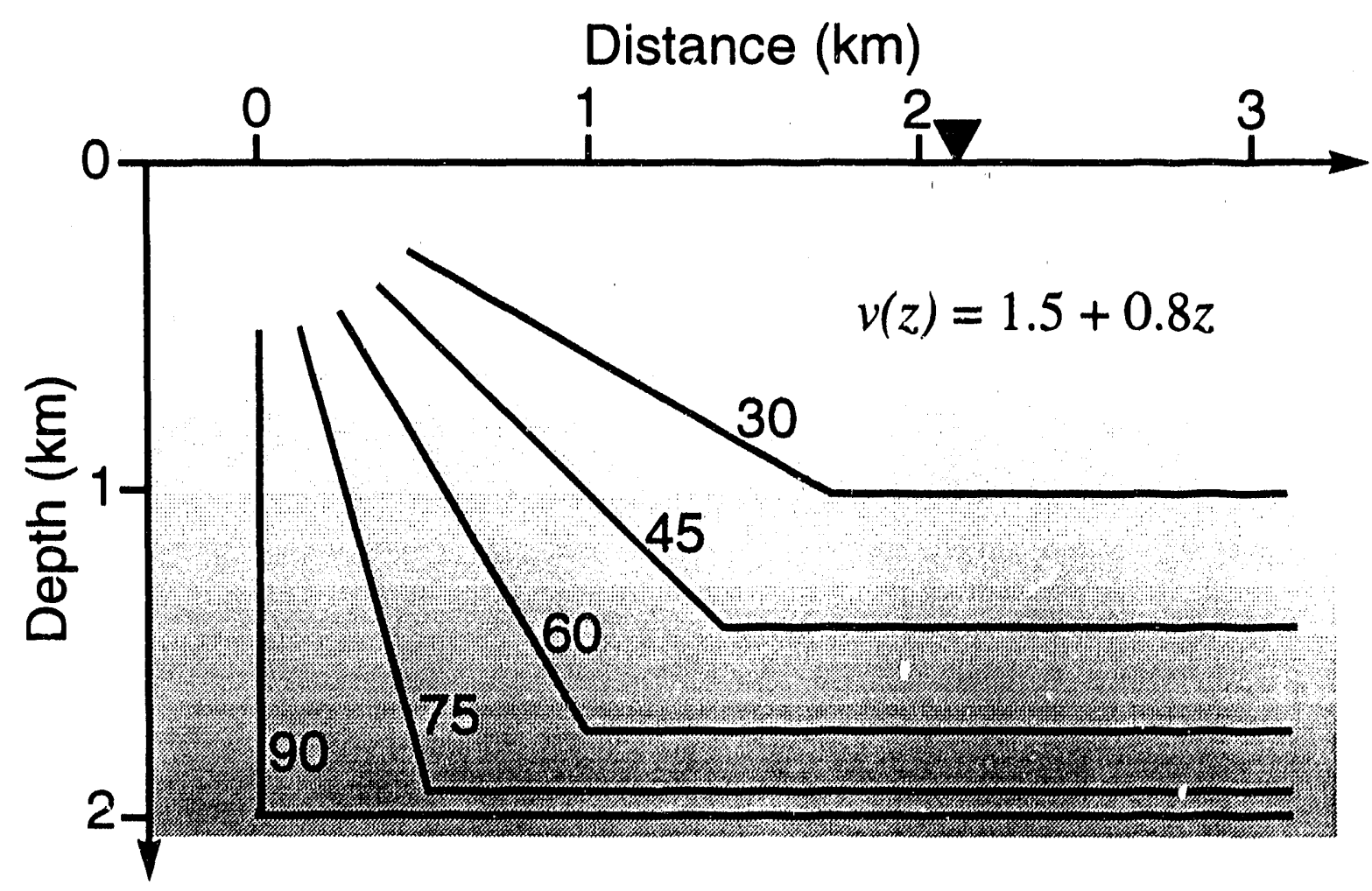

FIG. 3. Subsurface model used to generate synthetic seismic traces. The CMP gathers shown in Figure 4 corres'ond to the surface location marked here with a triangle. The five reflectors, each consisting of a dipping and horizontal segment, yield a total of ten reflections in each of those CMP gathers. 

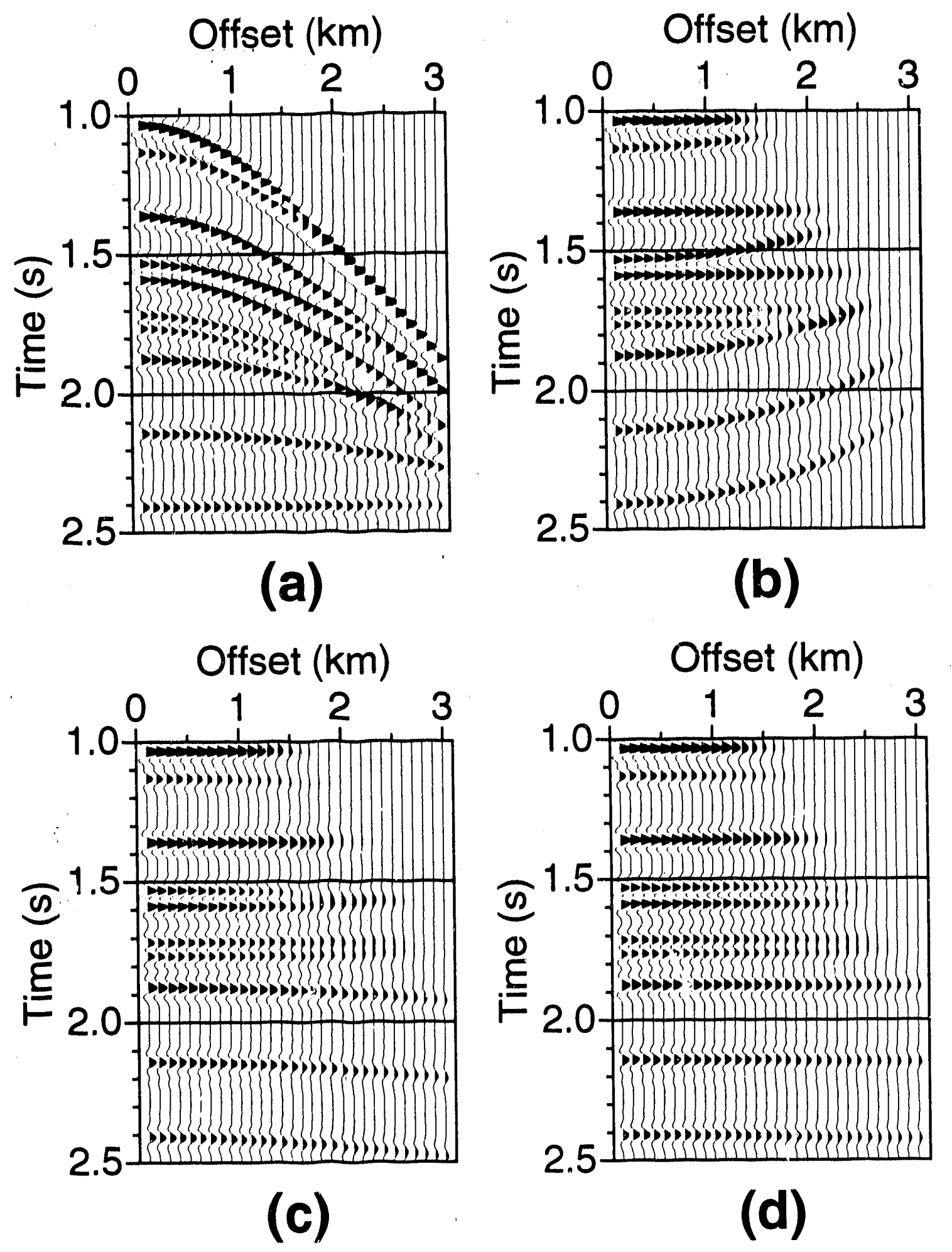

Fig. 4. (a) CMP gather for the model shown in Figure 3. (b) After NMO correction. (c) After constant-velocity DMO. (d) After squeezed DMO. The five reflections in (b) that are misaligned by NMO correspond to dipping reflectors. The last reflection in each gather corresponds to a dip of 90 degrees. 
for the steeper dips. In this test, squeezed DMO was performed using Notfors and Godfrey's (1987) DMO by Fourier transform, but with the modified time stretching defined by equation (11) and the modified phase-shifting factor of equation (12). As discussed above, we used a squeeze factor of $S \approx 0.4$ for this DMO method.

\section{RECORDED DATA EXAMPLE}

Like the synthetic data results of the previous section, the example of squeezed DMO applied to recorded data shown in Figure $1 \mathrm{~b}$ was obtained with our modified version of Notfors and Godfcy's Fourier transform method. In this example, howevir, we did not know a priori the velocity averages required to compute the squeeze function $\gamma(t)$. Therefore, we estimated the rms velocity function $V_{2}(t)$ by performing a typical NMO-based velocity analysis at several CMP locations. The resulting estimates of $V_{2}(t)$ were then averaged and smoothed to obtain the function plotted in Figure 5.

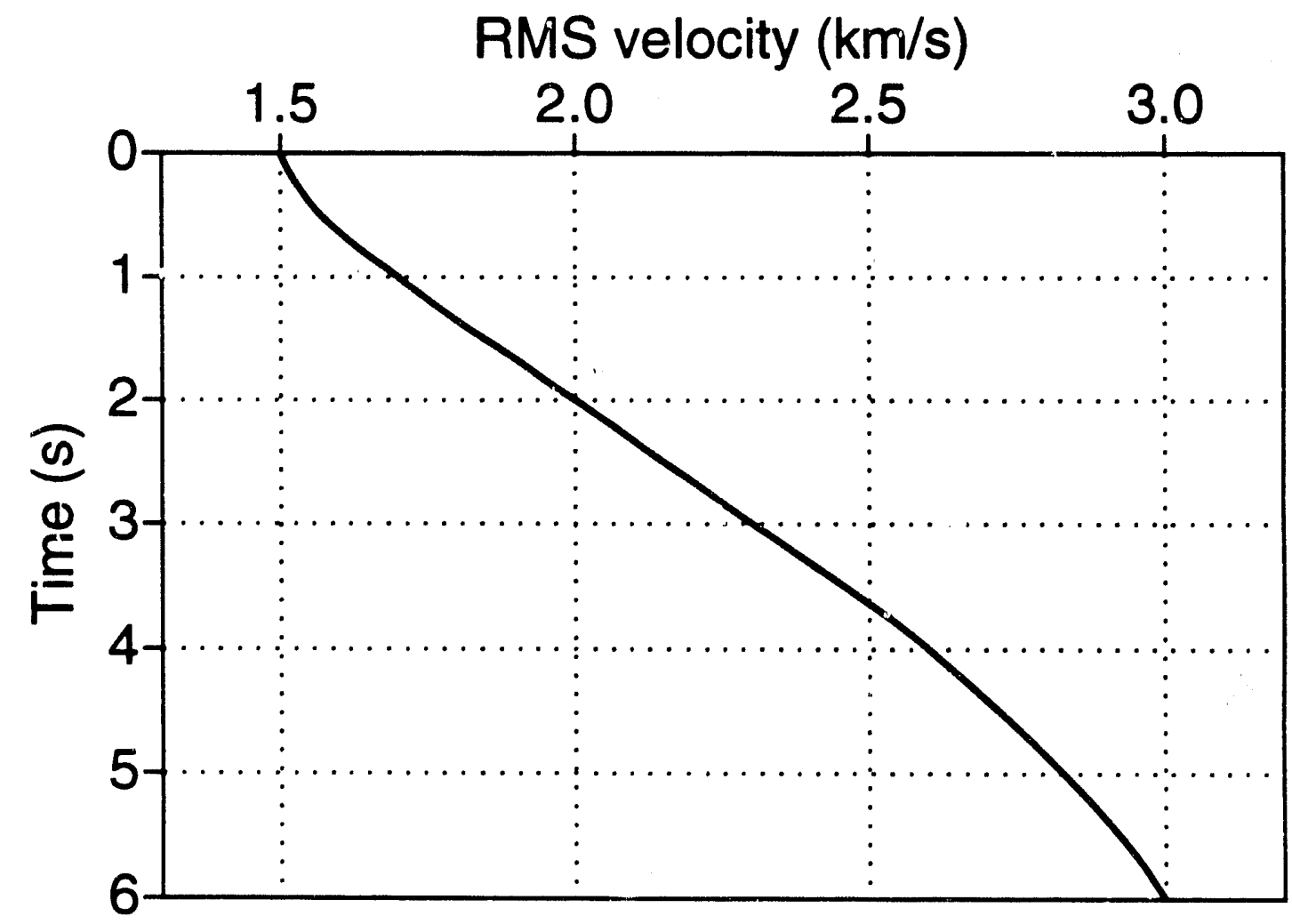

FIG. 5. The rms velocity function used to determine the squeeze function $\gamma(t)$ in DMO processing for the stack shown in Figure $1 \mathrm{~b}$.

We constrained the rms velocity function to be smooth for two reasons. First, the derivative with respect to time $t$ of $V_{2}(t)$ and, hence, $\gamma(t)$ in equation (3) should be continuous, in order for the squeezed DNO mapping to be continuous. Sccond, a 
more detailed velocity function would likely be inconsistent with the approximations used to derive equation (3). From this smooth estimate of $V_{2}(t)$, we computed the average $V_{4}(t)$ and the squeeze function $\gamma(t)$.

To determine those times for which squeezed DMO is most likely to yield significant improvement, we used the computed squeeze function $\gamma(t)$ and equations (4) and (5) to estimate the difference in the zero-offset times $t_{0}$ predicted by constant-velocity DMO and squeezed DMO. Assuming that this difference is sign: $:$ icant when it exceeds half the dominant period in the recorded data, we define significance of the squeeze function $\gamma(t)$ as the difference between squeezed DMO and constant-velocity DMO, expressed in units of half-cycle time shifts. Significance is plotted in Figure 6 as a function of time, for a reflection slope $\left(k / \omega_{0}\right)$ of $0.7 \mathrm{~s} / \mathrm{km}$, a source-receiver offset $(2 h)$ of $3 \mathrm{~km}$, and a dominant frequency of $30 \mathrm{~Hz}$. These parameters correspond roughly to the steep reflection in Figure 1 and to the length of the recording cable.

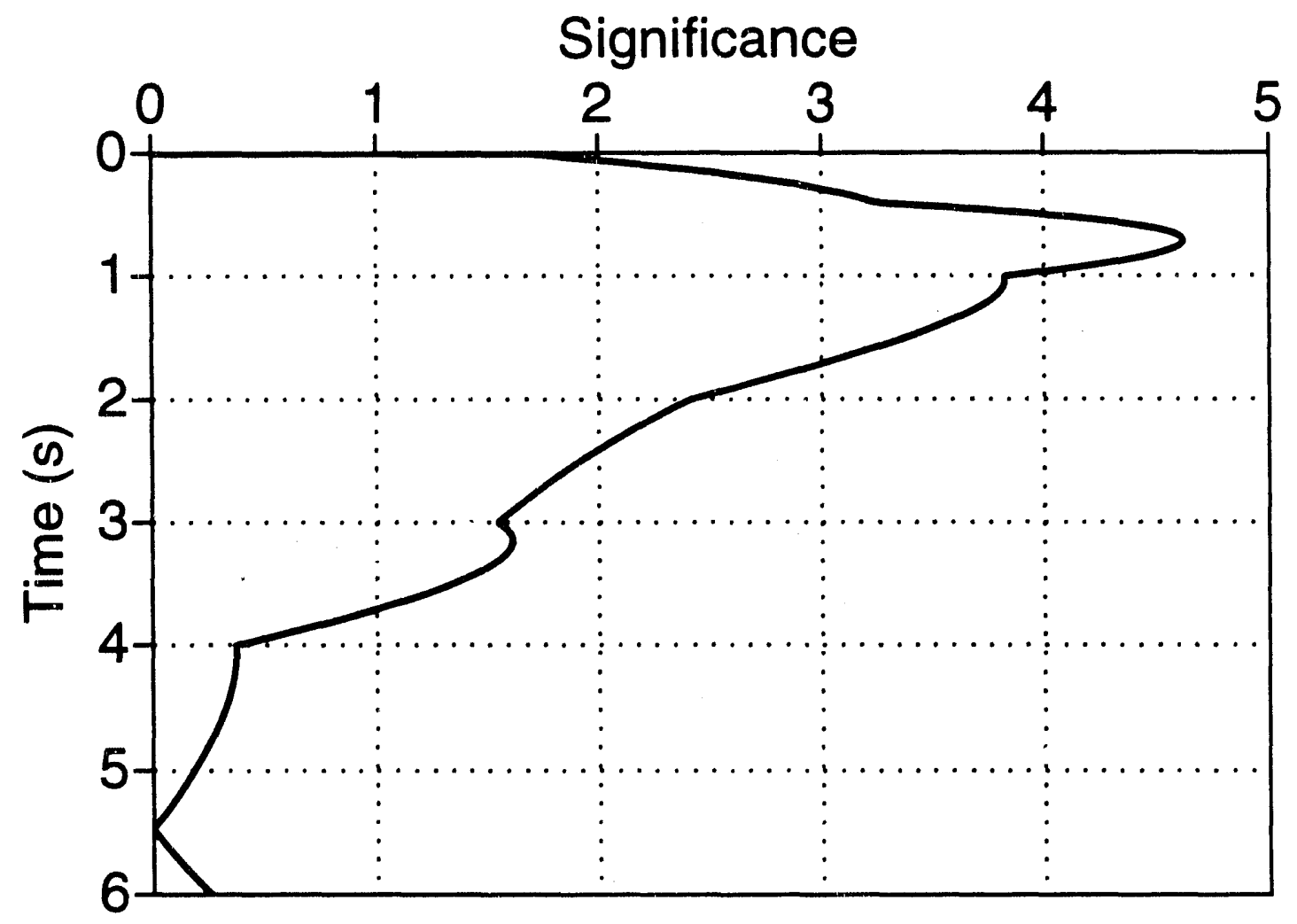

FIG. 6. Significance of the squeeze function $\gamma(t)$ as a function of time $t$, for the velocity function shown in Figure 5 , a reflection slope of $0.7 \mathrm{~s} / \mathrm{km}$, an offset of 3 $\mathrm{km}$, and a dominant frequency of $30 \mathrm{~Hz}$. The squeeze function is likely to be most significant for those reflections that just survive the mute, just below $2 \mathrm{~s}$ for a $3 \mathrm{~km}$ offset.

Ignoring times less than about $2 \mathrm{~s}$, which would typically be muted for an offset of $3 \mathrm{~km}$, Figure 6 shows that the squeeze function $\gamma(t)$ is most significant for times 
between 2 and $3.5 \mathrm{~s}$. We chose the range of times shown in Figure 1 specifically to highlight the differences between constant-velocity DMO and squeezed DMO where those differences are most significant.

The stack of the steep reflection in Figure la, obtained with constant-velocity DMO, could be improved by modifying the velocities used in NMO correction. Tests with the synthetic data illustrated in the example above suggest that decreasing the NMO velocities might compensate for the error in constant-velocity DMO. However, tunirg NMO velocities to account for errors in constant-velocity DMO may degrade the stack of horizontal reflections.

Figure 7 illustrates this point with a comparison of constant-velocity stacks computed with constant-velocity DMO and squeezed DMO. These stacks correspond to a small window extracted from the center of the stacks in Figure 1. With squeezed DMO (Figure $7 \mathrm{~b}$ ), both the horizontal and steep reflections at about $2.6 \mathrm{~s}$ are best stacked with a velocity somewhere between 2.10 and $2.15 \mathrm{~km} / \mathrm{s}$. With constantvelocity $D M O$, the steep reflections are best stacked with a lower velocity, between 2.00 and $2.05 \mathrm{~km} / \mathrm{s}$. In this example, squeezed DMO has enabled both the horizontal and steep reflections to be optimally stacked with the same NMO velocity.

The effect illustrated in Figure 7 may not always be consistent. Fur example, Lynn et al. (1991) suggest that ignoring anisotropy may, in some areas, compensate for the errors in constant-velocity DMO. In such areas, if we ignore anisotropy, we may observe that constant-velocity DMO enables both horizontal and steep reflections to be stacked with the same velocity, and that squeezed DMO does not.

\section{CONCLUSION}

Tests with both synthetic and recorded seismic data show that a simple squeezed version of constant-velocity DMO may be useful when imaging steep reflectors in a subsurface where velocity increases with depth. In the simplest implementation of squeezed DMO, we simply stretch time before and unstretch time after performing constant-velocity DMO. The time stretching function may be easily computed from a squeeze function $\gamma(t)$, which, in turn, depends on velocity averages that are likely to be available. To further improve squeezed DMO for steep dips, we tave also introduced a squeeze factor $S$, which requires only trivial and inexper sive modifications to constant-velocity DMO algorithms.

We were motivated to squeeze DMO by the surcess that others have had with time streiching in Stolt's (1978) constant-velocity migration, in order to approximately, but efficie: 'ily, handle velocity variations with depth. Because DMO correction generally moves seismic events less than migration, errors in DMO correction tend to be less significant than those in migration. Therefore. even where the errors in Stolt atretch migration are significant, we may expect squeezed DMO to provide an adequate approximation.

In this paper, we have omitted a thorough discussion of the differences between squeezed DMO and DMO methods that are exact for depth-variable velocity. This 


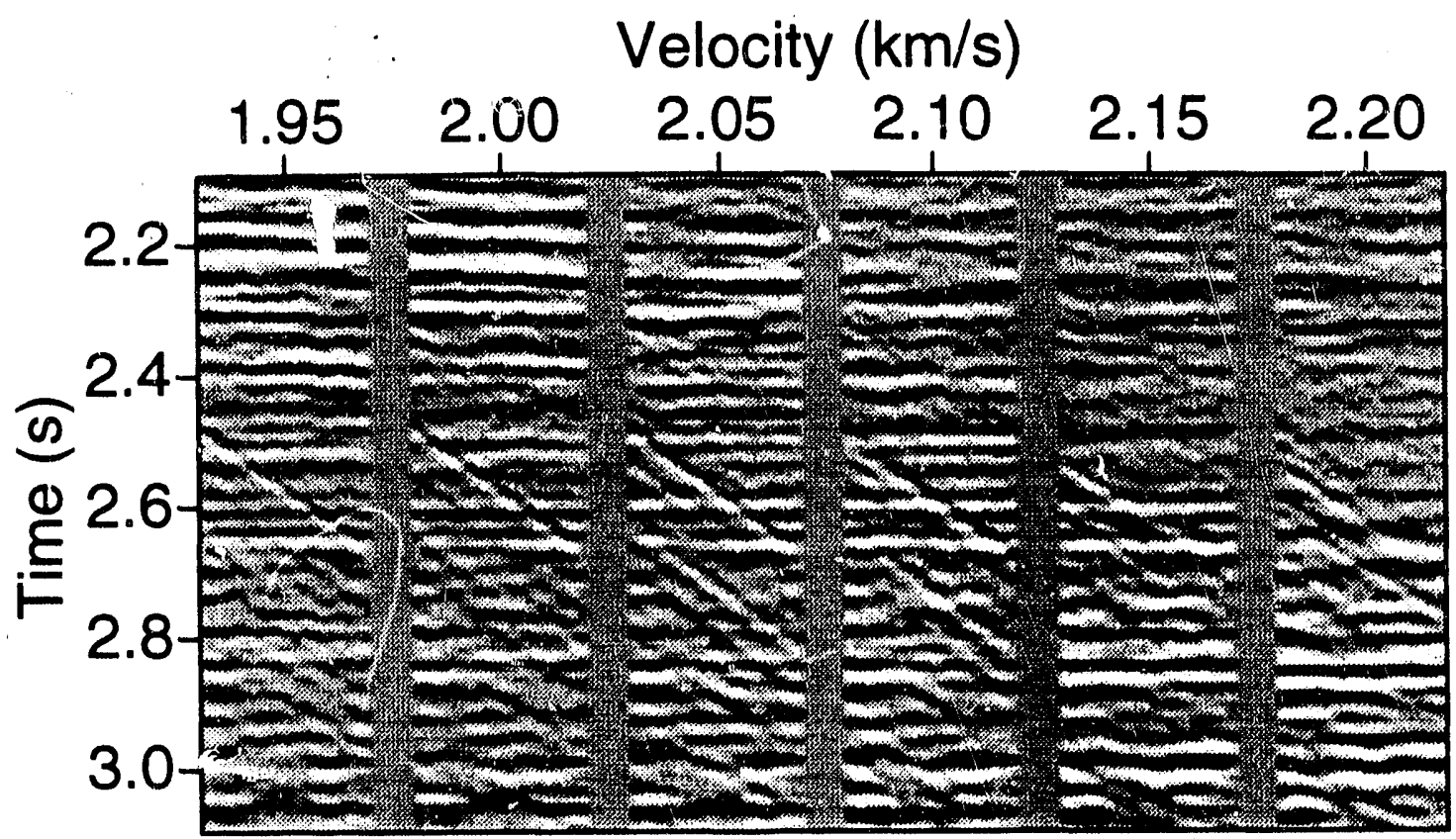

(a)

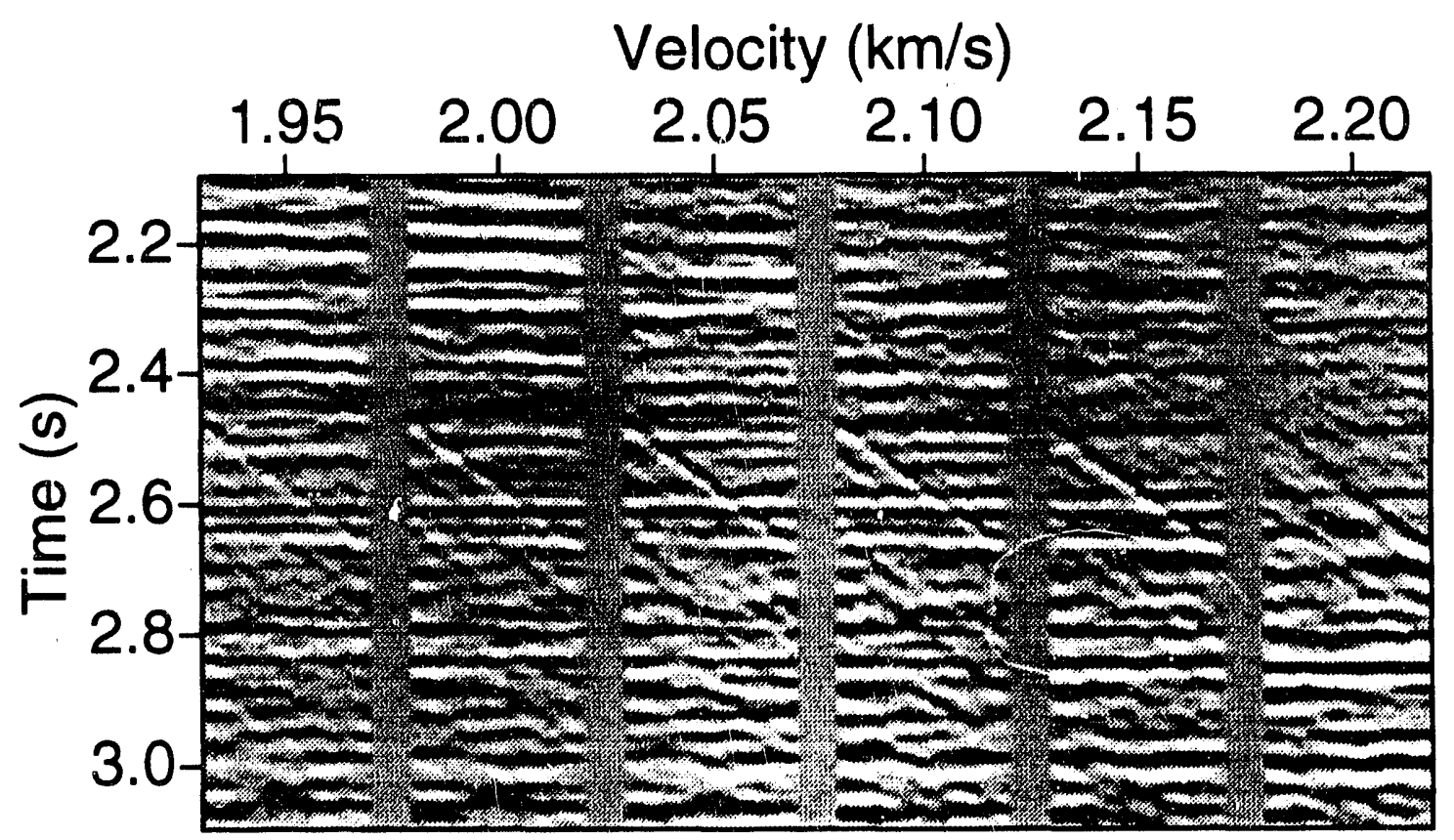

(b)

FIG. 7. Constant-velocity stacks computed after (a) constant-velocity DMO and (b) squeezed DMO. For each velocity, the stack shown here corresponds to a small window taken from the center of the corresponding section in Figure 1. With squeezed DMO, the steep reflection and the horizontal reflections at about $2.6 \mathrm{~s}$ are best stacked with the same velocity, between 2.10 and $2.15 \mathrm{~km} / \mathrm{s}$. 
discussion will be provided in a separate paper. One important difference that is too important to omit here is that squeezed DMO cannot account for crossline reflection point smear in 3-D seismic surveys. As shown by Perkins and French (1990), velocity variations with depth imply a $3-\mathrm{D}$ DMO operator that is not confined to lie in the inline plane containing the source and receiver locations. Time stretching before and unstretching after constant-velocity 3-D DMO cannot approximate the crossline component of the exact 3-D DMO operator.

\section{ACKNOWLEDGMENTS}

Fabio Rocca, while working with the Stanford Exploration Project years ago, laid the foundation for this work. The DMO squeeze factor $S$ was developed and tested through consultation with Advance Geophysical Corporation. Financial support for this work was also provided by the United States Department of Energy, Grant Number DE-FG02-89ER14079. (This support does not constitute an endorsement by DOE of the views expressed in this paper.) Additional support was provided by the members of the Consortium Project on Seisnic Inverse Methods for Complex Structures at the Center for Wave Phenomena, Colorado School of Mines.

\section{REFERENCES}

Artley, C. T., 1991, Dip-moveout processing for depth-variable velocity: Presented at the 61st Ann. Internat. Mtg., Soc. Expl. Geophys.

Barry, K. M., and Drecun, R. M., 1989, Some practical aspects of 3-D DMO: Presented at the 59th Ann. Internat. Mitg., Soc. Expl. Geophys.

Biondi, B., and Ronen, S., 1987, Dip moveout in shot profiles: Geophysics, 52, 1473-1482.

Bolondi, G., and Rocca, F., 1985, Normai moveout correction, offset continuation and prestack partial migration compared as prestack processes, in Fitch, A. A., Ed., Developments in geophysical exploration methods, 6: Elsevier Applied Science Publ., Ltd.

Deregowski. S. M., and Rocca, F., 1981, Geometrical optics and wave theory of constant offset sections in layered media: Geophys. Prosp., 29, 384-406.

Deregowski, S. M., 1985, An integral implementation of dip moveout: Presented at the 47th Mtg., Eur. Assin. Expl. Geophys.

Deregowski, S. M., 1986, What is DMO?: First Break, 5, no. 7, 7-24.

Deregowski, S. M., 1987, An integral implementation of dip moveout: Geophysical Trans. of the Geophys. Inst. of Hungary, 33, 11-22.

Haie, D., 1983, Dip moveout by Fourier transform: Ph.D. thesis, Stanford University.

Hale, D., 1988, Dip moveout processing, in Domenico, S. N., Ed., Course notes series, 4: Soc. Expl. Geophys.

Hale, D., 1991, A nonaliased integral method for dip moveout: Geophysics, 56. 795-805. 
Liner, C., 1990, General theory and comparative anatomy of dip moveout: Geophysics, 55, 595-607.

Lynn, W., Gonzalez, A, and MacKay, S., 1991, Where are the fault-plane reflections: Presented at the 61st Ann. Internat. Mtg., Soc. Expl. Geophys.

Meinardus, H. A., and Schleicher, K., 1991, 3-D time-variant dip-moveout by the FK method: Presented at the 61st Ann. Internat. Mtg., Soc. Expl. Geophys.

Notfors, C. D., and Godfrey, R. J., 1987, Dip moveout in the frequency-wavenumber domain: Geophysics, 52, 1718-1721.

Popovici, A M., 1990, Kinematics of prestack partial migration in variable velocity media: Presented at the 60th Ann. Internat. Mtg., Soc. Expl. Geophys.

Stolt, R. H., 1978, Migration by Fourier transform: Geophysics, 43, 23-48.

Witte, D., 1991, Dip moveout in vertically varying media: Presented at the 61 st Ann. Internat. Mtg., Soc. Expl. Geophys. 

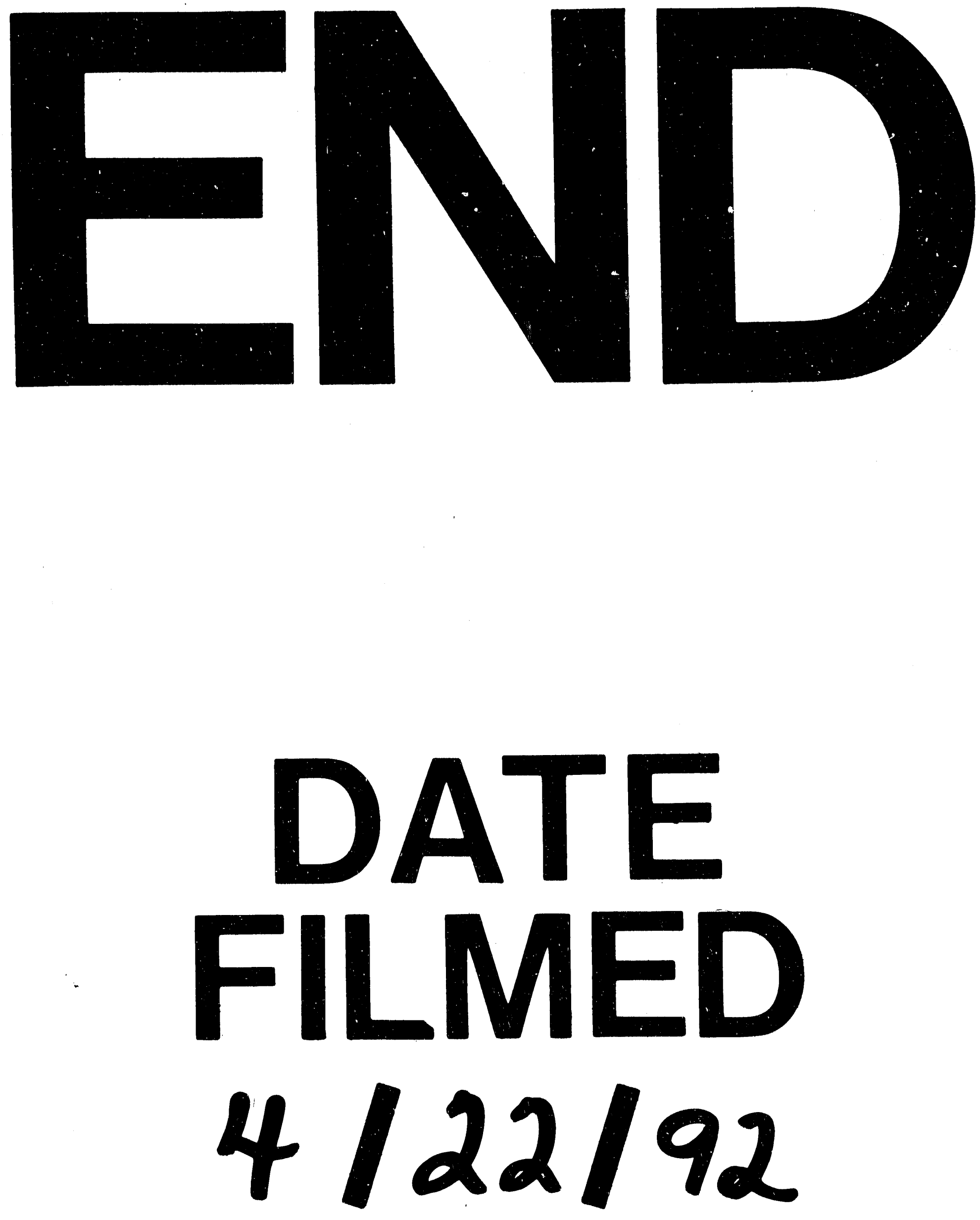
